\title{
Agenda for the 36th General Assembly of the European Association for the Study of Diabetes to be held in Jerusalem, Israel, in Hall A - Ussishkin, Wednesday, 20th September 2000 from 17.30-18.30 hours.
}

1. Minutes of the 35th General Assembly, Brussels, Belgium

2. Reports
a) President
Dr. J. Nerup
b) Honorary Treasurer
Dr. R. J. Heine
c) Honorary Auditors
Dr. R. Landgraf
Dr. J. Skrha
d) Honorary Secretary
Dr. M. Porta
e) Editor-in-Chief, Diabetologia
Dr. W. Waldhäusl

3. Elections
a) President 2001-2004
in place of Dr. J. Nerup (DK) retiring by rotation
b) Adjunct Honorary Secretary
Dr. A.J.M. Boulton (UK) 2000-2001
c) Editor-in-Chief, Diabetologia
Extension of term of office by 2 years
Dr. W. Waldhäusl (A)
d) Council Members 2000-2003
in place of
Dr. T. Halmos (H)
Dr. M. Hod (Israel)
Dr. D. Koev (Bulgaria)
Dr. I. Valverde (Spain)
4. Honorary Membership
Dr. D. Steiner (USA)

5. Any other business 